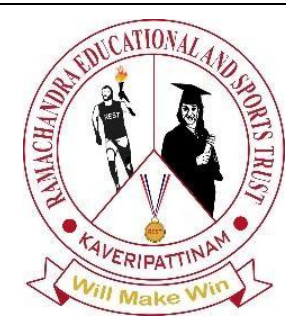

தமிழ் மொழி மற்றும் இலக்கியத்தின் சமகாலத்தன்மை

மலர் 2, இதழ் 1, 2021

ராமச்சந்திர கல்வி மற்றும் விளையாட்டு அறக்கட்டளை பதிப்பகம் பன்னாட்டுத் தரப்புத்தக எண்: 9788194845959

இணையதளம்: http://restpublisher.com/book-series/ctll/

\title{
இயக்குனர் சனு ராமசாமி தர்மதுரையில் முன்வைக்கும் அறம்
}

\author{
சா ஆரோக்கியம் \\ உதவி பேராசரியர், தமிழ் இலக்கியத்துறை \\ அருளானந்தர் கல்லூரி (தன்னாட்ச)
}

வாழ்வுக்கும் சாவுக்கும் புகழுக்குமான போராட்டத்தினுடே இருப்பை தக்கவைத்துக்கொள்ள ஓட வேண்டியதிருக்கிறது.அந்த ஓட்டமானது எதைமுன்னிருத்தியதாக இருக்க வேண்டும், எப்படி இருக்க வேண்டும் என்று பார்க்கிறபோது அது கேள்விக்குறியாக நிற்கிறது. ஏனெனில் நடைமுறை வாழ்வு என்பது கானல் நீர் போன்றதாகவும், மற்றவர்களுக்காக வாழ வேண்டிய நிர்பந்தங்கள் நிறைந்ததாகவும், பல நேரங்களில் ஓலமும், மௌனமும் நிறைந்ததாகவே அவ்வெளியை நிர்மானிக்க இடமி ருக்கிறது. இந்த புள்ளியிலிருந்து நோக்கும் கனம் வாழ்வு என்பது ஏற்கனவே தீமானங்களோடிஆனதாகஅல்லது அனுமானங்களோடு ஆனதா என்றால் அதற்கும் சரியான விடை இல்லை.ஏனென்றால் இரண்டுமே உணர்வுகளை சதைக்கக் கூடிய புள்ளிகள் தான். எப்படி என்றால் நான் அப்படி எண்ணினேன்! நான் இப்படி எண்ணினேன்!ஆனால் இது இப்படி ஆகும் என்று எண்ணவில்லை, என்கிற ஊசலாட்டத்தை தோற்றுவிக்கிறது. ஆக இந்த புள்ளியானது வாழ்வு அவ்வளவு சக்கரம் கடந்து செல்லக் கூடிய ஓன்று அல்ல நிறைவாழ்வு குறை வாழ்வு என்ற அளவுகோல் என்பது எதை வைத்து தீர்மானகிறது. என்றால் குறைந்தபட்ச அனுமானங்களோடு தான் அதாவது பொதுப்புத்தியிலிருந்து பார்க்கும் போது தான். ஆக வாழ்க்கை குறித்தான வெளியானது மேற்கண்ட நாண்களைக் கொண்டதாக காணப்பட்டு வருகிறது. அதே நேரத்தில் இதனுடே தான் வாழ வேண்டிய கட்டாயமிருக்கிறது. அத்தகு புள்ளிகள் பல நேரங்களில் பல்வேறு போக்குகளை, அனுமானங்களை முன் முடிவுகளை உடையதாக காணப்படுகிறதை உணரலாம். ஏன் அப்புள்ளிகளை இங்கு பேச வேண்டிய இருக்கிறென்றால் இப்படத்தல் வாழ்வின் போக்கானது அவ்வெளியில் பயணித்திருக்திறதாக அனுமானம் கொள்ளலாம். உலகம் முழுக்க கலைப்படைப்புகள் அறமென்ற ஒன்றை மையப்படுத்தியும், கடந்தும். சலாகித்து வந்திருக்கிற, வந்து கொண்டிருக்கிதை பார்க்கலாம் •அந்த அடிப்படையில் திரை ஊடகத்தின் வழி அறமென்ற ஒன்றை பல்வேறு கதை தளங்களில் கோணங்களில் சொல்லும் முறைகளின் நேர்துத்தியில் வித்தியாசங்களை செய்தி ருக்கின்ற ஆளுமைகளை பார்க்க முடிகிறது .காலவேட்டத்திற்கு ஏற்றாற்போல சொல்லும் முறைகளில் சமகால பிரஞ்றையோடு முன்னவப்புக்களை எடுத்து வைக்க வேண்டியதருக்கிறது. அதை எத்தகு பொறுப்புணர்வோடு முன்வைக்க வேண்டியது இருக்கிறது .என்று பார்க்கும் போது கலை படைப்பு களில் நடைமுறை வாழ்க்கையின் பிரதான வெளியை தத்ரூபமாக படம் பிடித்துக் காட்டுவதே கலையின் கடமையும், பொறுப்புமாகும். அந்த அடிப்படையில் வாழ்க்கைக்கான இருப்பை தக்கவைத்துக் கொள்வதற்கும் ,அதனூடாக விளையாடுகின்ற உணாவேட்டங்களை தேற்றிக் கொள்வதற்கும் மனம் போராடுகிற வெளியாக, சமூக வெளியை அனுமானிக்கலாம். இதில் கலைஞனை ஏன் காலம் கொண்டாடுகிறது என்றால் நமது வலியை அதாவது கடந்த ஏக்கத்தை ,மகிழ்ச்சயை அழுகையை, ஏகாந்த, உக்கிர,மௌனத்தை பெண்ணின் மொழி, பெண்ணின் உடல் மொழி என அனைத்து உணர்வுகனளயும் மனதிற்கு நெருக்கமாக வடிவமாக்க வேண்டியதுள்ளது. அப்போது அப்படைப்பு ஏதாவது ஒரு புள்ளியில் வாசகனிடத்தில் தனித்த இடம் பிடிக்க இடமிருக்கிறது. அந்த நோக்கல் சனு ராமசாமியின் தர்மதுரை படைப்பானது ஓரு தாகம் தீர்க்க இடைப்படுகிற ஓர் சுணையை போல அது வந்து போயிருக்கிற காலகட்டத்தை சொல்லலாம். இப்படைப்பு பின்பும் முன்பும் ஓரளவிற்கு அறத்தை முன்னிலைப்படுத்தி சல படைப்புகள் நகர்ந்திருக்கிறது. சம காலகட்ட கலை வெளியில் குறைந்தபட்ச எத்திக்ஸ் என்று சொல்லப்படுகிற அறச்சிந்தனை இருந்து வருகிறதா என்றால் அது கேள்விக்குறியாகும். அதே நேரத்தல் கலை மனமானது அறமனத்தோடு கூடியதே, ஆனால் அது குறைந்து அதற்கு மாறாக அதன் தன்மைகளை பயன்படுத்தி வருகிற போக்கிருக்கிறது, இது எப்படி என்பது புரியவில்லை. பாலுமகேந்திராவின் படங்களை பார்க்கின்றபோது அறநெறிக்கு சற்று பிறழ்ந்து அது இருக்கிறதா, அது அப்படியே சென்றாலும் அதில் ஓர் அறமி ருக்கிறதாகவே உணரலாம். அத்தகு பார்வையானது படைப்புல வெளியில் தேவையாக இருக்கிறது •

மையமாகக்கொண்டு ஒரு குடும்பத்திலுள்ள ஆண்களுக்கு பீமா, தர்மா எனவாறு பெயர் வைக்கப்பட்டிருக்கிறது. அவர்கள் ஒற்றுமையாகயிருக்க வேண்டுமென்பதற்காகவே பஞ்சபாண்டவர்கள் பெயர்களை வைக்கன்றனர். ஆனால் அவர்கள் அதற்கு மாறான சநந்தனை உடையவர்களாகவே சத்தரிக்கப்படுகின்றனர். எப்படி மகாபாரதத்திலிருந்த வினைனயப் போல இங்கு சீட்டு பிடித்தல் அதை தர்மா தவிர்க்க மறறற பாத்திரங்கள் செய்து வருகின்றன. இது தவறான அணுகுமுறை என நினைத்து அதை தர்மன் பிரகடனப்படுத்துகிறான். பின்பு அடைத்து வைக்கப்பட்டு வெளியூருக்கு களம்புகிறான். கிளம்பும் இரவில் சீட் பிடித்து வைக்கப்பட்திருக்கிற பணப்பையில் தெரியாமல் துணியை எடுத்து வைத்து களளம்பி விடுகிறார். கிளம்பி அவன் சந்திக்கிற நபர் மருத்துவம் படிக்கன்ற காலத்தில் தன்னை நேசித்த பெண்ணின் வீட்டிற்க்குச் செல்கிறார். அவ்விடம் கொடைக்கானலாக இருக்கிறது. அவ்விடத்தை இயக்குனர் காட்ச படுத்துகிறபோது "The Away Home" என்று சொல்லப்படுகிற கொரியன் படத்தில் படத்தின் கதாநாயகி, நாயகன் சந்தித்து கொள்கின்ற சமவெளிப் பகுதிகளின் Landescap மொழியை போல இக்காட்ச பதிவுகளை கொடுத்துருக்கிற கருதலாம். ஆனால் அதே நேரத்தல் அவள் இறந்து இருக்கிறார். அந்த இடத்திற்கு தேவைப்படுகிற வசனத்தை கையாண்டு ஓரு உயிர்ப்புடைய வெளியாக அதை காட்சப்படுத்தியிருக்கிறார். பின்பு சுபா என்று சொல்லப்படுகிற தன்னை நேசித்த அதை வெளிப்படுத்ததா பெண் அவரது வீட்டைத் தேடிச் செல்கிறான். அந்த தேடலிருந்து தான் தர்மதுரை படம் எழுச்சியுறுகிறதாக கருதலாம். ஏனெனில் அப் பெண் தனியாக ஒரு மருத்துவமனையை வைத்து மருத்துவம் செய்து வருகிறார். தருமணம் முடித்தும் தனித்து வாழ்ந்து வருபவளாக பார்க்க முடிதிறது. விவகாரத்துக்கு விண்ணப்பம் செய்திருக்கிறாள். இந்த சூழ்நிலையிலே கல்லூரித்தோழன் விஜய் சேதுபதி வருகை நிகழ்கிறது. அவனுடைய தோற்றப் பொழிவை பார்த்து இப்பொழுது 
சேவை செய்கிறாயா? இல்லையா? ஏன் என வினாவுகிறார். அப்போது கதாநாயகன் தன் ஊரில் உள்ள ஓர் காவல்காரர் மகள் மீது பிரியம் கொண்டு , பெண் கேட்டு திருமணம் வரை சென்று நின்று விடுகிறது. வரதட்சனண ஏதும் இல்லாமல் அவளது அன்பை மட்டுமே அவன் விரும்பி ஆசைப்படுகிறான். உடன்பிறந்த சகோதரர்கள் பணத்தற்கு ஆசைப்பட்டு அதிக தொகை அந்த ஏசை ஊர்காவல்காரரிடம் கேட்க, இயலா நிலை மகள் வருந்துகிறார். அந்த நிலையில் யார் காலில் விழுந்தாவது எப்படியாவது இதை செய்து வைக்க றேன் என கூறுகிறார். ஆனால் தன் அப்பா யார் காலிலும் விழுந்து சரமப்பட்டு இந்த திருமணத்தை நடத்தக் கூடாது என்று மருந்து குடித்து இறந்து விடுகிறாள். இப்படத்தில் இரு துருவங்கனள முன்னிலைப்படுத்துகிற புள்ளியை பார்க்கலாம். அதாவது ஏற்கனவே திருமணம் முடிந்து அவனோடு) வாழ பிடிக்காமல் விவாகரத்து விண்ணப்பித்து, தனித்து வாழ்ந்து வருகிற பெண்மணி , மற்றொன்று கதாநாயகன் ஆசைப்பட்டு பெண் கேட்டு திருமணம் வரை சென்று வரதட்சணை என்ற ஒரு புள்ளியில் இறந்து போகிற ஓரு பெண்மணி ஆக இந்த இரண்டு பெண்மணிகளின் வாழ்வும் கேள்விக்குறியாய் போன ஓர் இடத்தை உணரலாம். இதற்கடையே கதாநாயகன் சுபா வீட்டிலே தங்குகிறான். அவள் விவகாரத்திற்கு விண்ணப்பித்து இருக்கிறார் • ஓர் புள்ளியில் அது நெருக்கமான ஓரு சூழலுக்கு இட்டுச் செல்கிறது, அதே நேரத்தில் விவகாரத்து தீர்ப்பும் வருகிறது. அப்பொழுது கதாநாயகன் சுபாவின் முழு விபரத்தை அறிகிறான். இயக்குனர் ஓரே ஓர் வசனத்தல் அத்தனையையும் சொல்லியிருப்பதாக, ஓரு காட்சயில் நான் என் அன்புசெல்வியா உன்னை பார்க்கிறேன் என்கிறார். பின்பு பிரியங்கள் இரண்டும் சங்கமமாகின்றன. இப்புள்ளியானது மிக முக்கியமானதாக உணர முடிகிறது. ஏன்னெனில் கதாநாயகி சுபா கருவுற்று அது பிடிக்கவில்லையென அந்த கருவை கலைத்து விடுகிறான். ஆகவே அந்த மிருகத்தோடு வாழ விருப்பமில்லாது விவகாரத்து செய்து தனித்து வாழ்கிற அத்தகு பெண்மணி கதாநாயகனை வகுப்பு தேர தன ஏற்றுக்கொண்டு வாழ்கிற புள்ளி என்பது அறமென்ற ஒன்றேயாகும். இருவருக்கும் இடையே ஓர் அறமிருக்கிறது அந்த அறமானது, இருவரையும் இணைத்து இருப்பதாக எண்ணலாம். உலகம் முழுக்க அந்த அறம் தான் அனைவரையும் வாழவைக்கிறது. இங்கு இரு வேறு துருவப் பின்னணி உடைய உயிரிகளை இளைத்து வாழ வைத்திருக்கிற புள்ளி என்பது எது என்றால் அது அறமயிருக்கிறது குறிப்பிடத்தக்கது. அந்த அறமானது தனிமனித ஒழுங்கில் ஒவ்வொருவருக்கும் தேவையாக இருக்கிறனத பிரகடனபடுத்துகிறதாக உணரலாம். அத்தகு புள்ளியிலேயே கதாநாயகன் தர்மத்தின் துரையாக நிற்கிறனத கானலாம். இதற்கடையே மூன்று கதாநாயகிகளை கதையில் கொண்டு மூன்று வெவ்வேறு வாழ்வியல் பிம்பங்களையும், சக்கல்களையும் கொண்டதாக சொல்லி நடைமுறை வாழ்வில் பெண்கள் இத்தகு புள்ளிகளை எல்லாம் கடந்தே வாழ வேண்டிய தேவையிருக்கிறது என்பதை சொல்லியிருப்பதாக விளங்கலாம். அதை ஒவ்வொரு காட்சயிலும் மிக நேர்த்தியாக அழகியல் உணர்ச்சி ததும்ப, நிறைந்து அதை காட்சயயாக்கியிருப்பது இனிக்கிறது• அதே நேரத்தில் ஒரு பக்கம் மௌனமாயி ருக்கிறதையும் சொல்லியாக வேண்டியதிருக்கிறது. ஒரு தேர்ந்த உண்மையான படைப்பாளி என்ற அடிப்படையில் சமூக அக்கறையுடனான பல்வேறு விஷயங்களை சொல்லியிருக்கிறதாக கருதலாம். உதாரணமாக திருநங்கை குறித்தான காட்ச வருகிறபோது படத்தில் முதன் முதலாக அவரை காட்டுகிற போது ஒரு வீட்டை பார்த்துக் கொண்டிருக்கிற வீட்டு காவலாளி பெண்ணாக காட்டுகிறார். அடுத்து கதாநாயகனின் மருத்துவமனையில் வேலை செய்கிற பெண்ணாக காட்டுகிறார். வர்களது குரலுக்கு ஆதரவு குரல் கொடுத்தருக்கிற ஓர் படைப்பாக முன் வைத்து இருப்பது சறப்பு. பாடல்களை பேசயயாக வேண்டிய தேவை இருக்கிறது •ஏனெனில் தென் மாவட்டத்தின் குறிப்பாக மதுரையைச் சுற்றியுள்ள பகுதி மக்களின் வாழ்வியலை அப்படியே படம் பிடித்துக் காட்டியிருப்பது

$$
\begin{aligned}
& \text { ஆண்டிபட்டி கனவா காத்து } \\
& \text { ஆள தூக்குதே } \\
& \text { அத்த பொண்ணு } \\
& \text { என்ன தாக்குது } \\
& \text { அட முட்ட பொம்பளையே } \\
& \text { என்ன முழுசா } \\
& \text { நம்பளையே உனக்கும் } \\
& \text { உன் பதவிக்கும் } \\
& \text { வெள்ளரிக்காய் பிடிப்பா } \\
& \text { இந்தக் சிறுகிய } \\
& \text { ஏலச் ஓறுக்கிய } \\
& \text { எதுக்காக புடிச்ச } \\
& \text { ஓரு வெள்ளகரரி } \\
& \text { காசு தீர்ந்தா வெறுத்து } \\
& \text { ஓடிப்வோவது } \\
& \text { இவ வெள்ளரிக்கா } \\
& \text { வித்து கூட } \\
& \text { வீடு காத்து வாழ்வா" }
\end{aligned}
$$

என வருகின்ற பாடல் படத்தின் கதை கருவுக்கு ஒத்து மிக நேர்த்தியாக பாடல்காட்ச , இசை உருவாக்கம் பிரதான வலு சேர்த்தாலும் பாடல் வரிகள் அதற்கு மேலும் வலு சேர்ப்பதாக எண்ணலாம். அதே போன்றே அனைத்து பாடல்களும் இன்னொரு பாடல்கூட கட்டாரில் தொடங்கும் கலக்கல் மென்மை மொழியாக

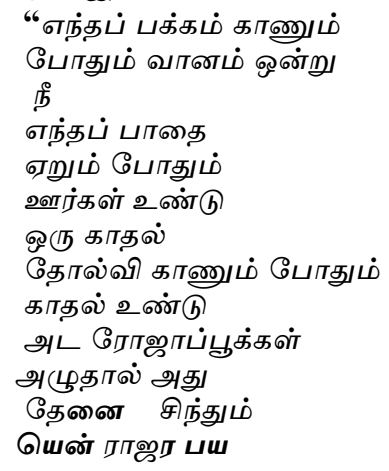


நீ அழுதால்

அதில் யானம் மிஞ்சும்

உன் சோகம் என"

உள்ளத்தை வருடுகின்றதை உணரலாம் • இந்தப்பாடலில் பாடலாசிரியர் அவர்கள் கதாநாயகனை ஒரு ராஜாவாக ,ஒரு ஞானியாக பார்த்தருக்கன்ற தன்மையை கிரக்க முடிகிறது. இப்படியாக பாடல்காட்சககளில் மதுரை மண்ணின் மொழியை தத்ரூபமாக , காட்சப்படுத்தி மனதிற்கு நெருக்கமான ஒரு கலைப்படைப்பாக காட்சிகளை முன்வைத்திருக்கிறார். மிகுந்த நேர்த்தியான காட்சகளில் ஒரு காட்ச்ப் பதிவு அதாவது தேனி மாவட்டம் ஆண்டிப்பட்டி கணவாயை கடக்கிற அனைத்து ஜீவன்களும் உணர்கிற உணர்வாகும். ட்ரோன் ஷாட்டில் அப் பாடலை அமைந்து ஓரு கவிதை இன்னொரு கவிதை மொழியை பேசுவதை போல அமைத்திருப்பதில் அழகியல் உணர்வு ததும்புகிறது. குறிப்பிடத்தக்கது இன்னொரு பாடல் நாட்டுப்புற சாயலில் அமைந்த பாடல் அப்பாடல் தொடங்கும் போதே பேசப்படுகிற வசனம் கவனிக்கத்தக்கது. அதாவது கலைஞனை ஒரு கலைஞனை யாராக இருந்தாலும் மதிக்க வேண்டும். என்ற அறத்தை பேசுதிற வசனமாகும். அத்தகு இடங்களில் கதாநாயகனுக்கு மக்களின் மனதில் தனித்த இடத்தை ஏற்படுத்தி தந்திருகின்ற ஒரு புள்ளியாக அப் பதிவை அனுமானிக்க முடிகிறது. இப்படத்தில் குறிப்பிட்டு பேச வேண்டிய ஓரு இடம் காமக்காபட்டி அன்பு செல்வி என்ற பெயரில் கதாநாயகன் விரும்பும் முதல் கதாநாயகி துணுக்குச் செய்திகள் எழுதி வருகின்ற பாத்திரப் படைப்பு, அதை நகர்த்தி இருக்கின்ற காட்ச பதிவுகளும் நினைவலைகளில் படிந்து கிடக்கும் காட்சிகளாக உணரலாம். பொதுவாக அங்கொன்றும் இங் கொன்றுமாக பெண்கள் நகரம் கிராமம் என இவ்வெளிகளில் இருக்கின்ற நூலகம் சென்று புத்தகங்களை தொடர்ச்சியாக எடுத்து படித்து வருகின்ற பெண்களை பார்க்கலாம். அதுபோலவே இதழ்களுக்கு துணுக்குச்செய்தி எழுதிவருகின்ற நபர்களையும் நினைவில் கொள்ளலாம்.இத்தகு புள்ளிகளெல்லாம் பெண்களில் எதாவது ஒரு புள்ளியில் தங்களைத் தகவமைத்துக் கொள்வதற்காக அல்லது அறநெறியில் நிற்க அவ்வெளி அனுபவங்கள் அவர்களுக்கு துணை புரிவதாக கருதலாம். அவ்வாறு பெண்ணின் மொழியையும் பேசயிருக்கிற படைப்பாக இப்படைப்டை அடையாளப்படுத்தலாம். காமராஜ் வாத்தியார் பாத்திரம் அறத்தை போதிக்கும் ஒரு முழுமையான பாத்திரமாக படைத்திருக்கிறார். ஒரு காட்சயில் கதாநாயகனை பார்க்க வருதிறார். அப்பொழுது அவருக்கு பார்வை தெரியாத நிலையில் கதாநாயகனை பார்க் வருகிறார். மருத்துவமனைக்கு வந்து மருத்துவரை பார்க்க வேண்டுமென்று வேலை பெண்ணிடம் சொல்லுகிறார். அவரும் போய் சொல்ல ஓடோடி வருகிறார். வந்து அவரின் காலில் விழுந்து வணங்க அந்த தொடுதலிலே அது தர்மதுரை தான் என்பதை உணர்கிறார். இத்தகு பதிவுகளில் படைப்பின் உணாவோட்ட நாண் மிகுந்து காணப்படுவதாக கருதலாம். மூன்று தன்டைகளை உடைய அன்பு உணர்வுகள் அதாவது அது சகோதரத்துவத்தையும், இசமத்துவத்தையும் ,முற்போக்கு சந்தனைகளையும் பேசுவதைக் காணலாம். இஸ்லாமிய கதாபாத்திரவெளி, கிறிஸ்துவ கதாபாத்திர வெளி,இந்து கதாபாத்திர வெளி என மூன்றையும் ஒற்றுமைப் படுத்தருக்கிறபுள்ளி, சங்கமிக்க வைத்திருக்கிற புள்ளி என இயக்குனரின் அனுபவ வெளிஆளுமைக்கு எடுத்துக்காட்டுகளாக அமைந்து மெருகூட்டுகின்றன. ஒட்டு மொத்ததில் அறமென்ற ஒரு புள்ளியில் அவையாவும் இணைகிறததாக கருத இடமிருக்கிறது. அற மழையை காட்சிகளில் நனைய விட்டிருக்கிற பதிவு வரவேற்கதக்கது. அறசிந்தனை அறவே கேள்விக்குறியாகி வருகிறது, கலைப் படைப்புகளில் குறிப்பாக திரை ஊடகத்தில் இத் தரைப்படமானது அதை மையப்படுத்தியே நகர்ந்து தனி மனித அறம் கலையின் அறம் கலையின் அறமே தனி மனித அறம் என்ற உயரிய சிந்தனையை போதித்தி ருக்கிற படைப்பாக இத்திை மொழியை விளங்கலாம். 